\title{
Enzyme replacement therapy for congenital hypophosphatasia allows for surgical treatment of related complex craniosynostosis: a case series
}

\author{
Libby Kosnik-Infinger, MD, MPH, ${ }^{1}$ Craig Gendron, MD, ${ }^{2}$ Christopher B. Gordon, MD, ${ }^{2}$ \\ Brian S. Pan, MD, ${ }^{2}$ John A. van Aalst, MD, ${ }^{2}$ and Timothy W. Vogel, MD ${ }^{1}$ \\ Divisions of ${ }^{1}$ Pediatric Neurosurgery and ${ }^{2}$ Plastic Surgery, Cincinnati Children's Hospital Medical Center, Cincinnati, Ohio
}

Hypophosphatasia (HPP) is a rare inherited disorder of bone metabolism that results in the loss of function of the gene coding for tissue-nonspecific alkaline phosphatase (TNSALP). Patients with HPP have defective bone mineralization as well as craniosynostosis that can be seen in the infantile and childhood forms of this disease. Traditionally, HPP has had a poor prognosis, with few children surviving to exhibit the phenotype of clinical craniosynostosis that requires surgical intervention. Here, the authors report on new advancements in enzyme replacement therapy (ERT) for children affected by HPP, allowing these patients to survive and undergo surgery to address complex craniosynostosis. The authors discuss their case series of 4 HPP patients treated at their institution with ERT who have undergone successful surgical intervention for craniosynostosis. These children had no complications related to their surgeries and exhibited decreased neurological symptoms following cranial vault remodeling. This study reveals that ERT administered either pre- or postoperatively paired with cranial vault remodeling strategies can yield improved neurological outcomes in children affected by HPP.

http://thejns.org/doi/abs/10.3171/2015.2.FOCUS14847

KEY WORDS hypophosphatasia; HPP; ENB-0040; asfotase alfa; craniosynostosis; pediatrics; craniofacial

$\mathrm{H}$ YPOPHOSPHATASIA (HPP) is a rare inherited disorder of bone metabolism with a live birth incidence of 1:100,000 in the general population. ${ }^{11}$ The disorder results from loss-of-function mutations in the gene coding for the tissue-nonspecific alkaline phosphatase (TNSALP) found on 1p36.411 These mutations alter the function of the serum and bone alkaline phosphatase activity, resulting in defective bone and teeth mineralization. ${ }^{13}$ Natural substrates of TNSALP accumulate, including inorganic pyrophosphate, an inhibitor of bone mineralization, and pyridoxal 5'-phosphate (PLP), the circulating form of vitamin B6. High extracellular levels of these materials block hydroxyapatite crystal growth and cause osteomalacia. ${ }^{7}$ In severely affected patients, hypercalcemia and hyperphosphatemia can develop. ${ }^{15}$ Most patients present with skeletal changes, short stature, painful lower limbs, gait disturbance, and premature shedding of teeth. ${ }^{4}$ Bones of the cranium can also be affected, resulting in complex forms of craniosynostosis..$^{4,11}$

The phenotypic expression of HPP is highly variable. Cases range from stillbirth to those with minor dental problems exhibited in adulthood. Because of this high variability, HPP has been classified into clinical subtypes according to the age of the patient when signs and symptoms first manifest (perinatal, infantile, childhood, and adult).,11 There are also 2 additional subtypes (odontoHPP and perinatal benign-HPP), which are less severe and do not present with craniosynostosis (Table 1). Perinatal HPP is diagnosed on prenatal imaging by severe generalized osteopenia and a nonossified cranial vault and is often lethal., ${ }^{4,6}$ Those neonates who survive often die shortly after birth due to respiratory compromise arising from hypoplastic skeletal deformities of the chest. ${ }^{11}$ Infantile HPP presents before 6 months of age with severe rachitic skel-

ABBREVIATIONS ERT = enzyme replacement therapy; HPP = hypophosphatasia; ICP = intracranial pressure; PLP = pyridoxal 5'-phosphate; TNSALP = tissue-nonspecific alkaline phosphatase.

SUBMITTED December 29, 2014. ACCEPTED February 12, 2015.

INCLUDE WHEN CITING DOI: 10.3171/2015.2.FOCUS14847.

DISCLOSURE The authors report no conflict of interest concerning the materials or methods used in this study or the findings specified in this paper. 
TABLE 1. The 6 clinical forms of hypophosphatasia*

\begin{tabular}{|c|c|c|c|c|}
\hline Clinical Form & Inheritance & Bone Symptoms & Dental Symptoms & Clinical Diagnosis \\
\hline Perinatal lethal & AR & $\begin{array}{l}\text { Hypomineralization, osteochondral } \\
\text { spurs }\end{array}$ & NA & Radiographs, ultrasonography \\
\hline Perinatal benign & $A D$ & $\begin{array}{l}\text { Bowing of long bones, benign } \\
\text { post-natal }\end{array}$ & NA & Ultrasonography, clinical examination \\
\hline Infantile & AR & $\begin{array}{l}\text { Craniosynostosis, hypomineraliza- } \\
\text { tion, rachitic ribs, hypercalciuria }\end{array}$ & Premature loss of deciduous teeth & $\begin{array}{c}\text { Clinical examination, biology (serum AP } \\
\text { activity, PEA and PLP), radiographs }\end{array}$ \\
\hline Childhood & $\begin{array}{l}\text { AR (frequent) } \\
\quad \text { or } A D \text { (rare) }\end{array}$ & $\begin{array}{l}\text { Short stature, skeletal deformity, } \\
\text { craniosynostosis, waddling gait, } \\
\text { bone pain/fractures }\end{array}$ & Premature loss of deciduous teeth & \\
\hline Adult & $A R$ or $A D$ & $\begin{array}{l}\text { Stress fractures: metatarsal or } \\
\text { tibia; osteoarthritis }\end{array}$ & $+/-$ & \\
\hline $\begin{array}{l}\text { Odontohypo- } \\
\text { phosphatasia }\end{array}$ & $A R$ or $A D$ & Loss of alveolar bone & $\begin{array}{l}\text { Exfoliation (incisors), reduced thick- } \\
\text { ness of the dentin, enlarged pulp } \\
\text { chambers of teeth, dental caries }\end{array}$ & $\begin{array}{l}\text { Clinical examination, biology (serum AP } \\
\text { activity, PEA and PLP) }\end{array}$ \\
\hline
\end{tabular}

$\mathrm{AD}=$ autosomal dominant; $\mathrm{AP}=$ alkaline phosphatase $; \mathrm{AR}=$ autosomal recessive; $\mathrm{NA}=$ not applicable; $\mathrm{PEA}=$ phosphoethanolamine; $\mathrm{PLP}=$ pyridoxal $5^{\prime}$-phosphate.

* Reproduced from Mornet E: Hypophosphatasia. Orphanet J Rare Dis 2:40, 2007. With permission from BioMed Central, copyright CC-BY-NC 2.0 (http://

creativecommons.org/licenses/by/2.0).

etal deformities, seizures, ${ }^{1,9}$ and premature fusion of the cranial sutures. ${ }^{4}$ Childhood HPP has variable expressivity and exhibits growth retardation, rachitic osteopathy, premature loss of deciduous teeth, and craniosynostosis.,11 Adult HPP is usually recognized during middle age, with patients having a prior history of rickets or early loss of teeth. Skeletal changes include short stature, numerous fractures, chronic pain, and loss of ambulation. ${ }^{4,17}$

Craniosynostosis is considered a feature of the infantile and childhood forms of HPP, and there are limited reports of craniosynostosis associated with HPP in the scientific literature. ${ }^{13}$ In an attempt to discover a phenotype-genotype correlation in HPP, there is a previous report of biochemical comparison of cases of childhood HPP with and without craniosynostosis. The results of that study did not reveal biochemical or a distinct genetic pattern that differentiated the 2 groups. ${ }^{4}$ Recent developments in enzyme replacement therapy (ERT) have allowed for improved treatment of children affected by HPP, thus making surgery for craniosynostosis a possibility. Late clinical sequelae such as increased intracranial pressure (ICP) resulting in papilledema can be addressed and avoided by surgically treating HPP patients who have craniosynostosis.

In this article, we describe our experience with the treatment of HPP and its associated craniosynostosis. We have treated 4 pediatric patients affected by HPP and craniosynostosis and present 2 representative cases diagnosed at Cincinnati Children's Hospital. All of these cases have been treated with ERT (Table 2).

\section{Case Series}

We have treated 4 pediatric patients with HPP and its associated craniosynostosis at our institution. The average age at presentation to our craniofacial team is 38.2 months. Each patient was treated with ERT according to an ongoing, approved clinical trial with agent ENB-0040 (asfotase alfa, Alexion Pharmaceuticals; Table 2). One patient received the treatment preoperatively and 3 received it postoperatively. All 4 patients presented with altered neurological function and have undergone neurodiagnostic imaging to confirm the presence of craniosynostosis.

Our 2 representative cases are from the same Saudi Arabian family, both having infantile HPP associated with craniosynostosis. There is a history of consanguinity in this family with 4 male cousins affected by HPP.

\section{Case 1}

The first patient was a 3-year-and-9-month-old boy who initially presented to the endocrinology service at the age of 2 years and 3 months. His medical history included HPP, craniosynostosis, restrictive lung disease, respiratory infections, failure to thrive, and multiple fractures. He was admitted to the hospital for management of acute exacerbation of a chronic respiratory illness. Consultation with our craniofacial team was requested to evaluate the clinical findings of dolichocephaly and ridging over the left coronal suture. The patient also presented with irritability, intermittent emesis and headaches, an asymmetrical cranial base, facial scoliosis, and bilateral papilledema. As part of a clinical trial, the patient was awaiting ERT with agent ENB-0040 (asfotase alfa).

Diagnostic radiographic imaging with 3D head CT revealed left coronal and sagittal craniosynostosis, calvarial thinning, dysplasia of the right frontal lobe, and venous anomalies including congenital absence of the right sigmoid sinus (Fig. 1). As a result of these findings, a frontoorbital advancement with cranial vault reconstruction was performed to reduce suspected intracranial hypertension as well as to correct the anatomical deformity. Allograft material (Grafton DBM in Flex and Putty forms, BioHorizons IPH, Inc.) was used to augment bone formation. The patient tolerated the procedure well with no complications noted.

Three weeks after surgery the patient was started on the study drug asfotase alfa at a dosage of $1 \mathrm{mg} / \mathrm{kg}$ given intramuscularly 6 times a week. The dosage was adjusted based on the study protocol. One year after the surgery, the patient was well with resolution of his papilledema and 
TABLE 2. Clinical summary of HPP cases treated

\begin{tabular}{|c|c|c|c|c|c|c|c|}
\hline $\begin{array}{l}\text { Case } \\
\text { No. }\end{array}$ & Age (yrs), Sex & $\begin{array}{l}\text { Neurological Signs/ } \\
\text { Symptoms }\end{array}$ & $\begin{array}{l}\text { Imaging } \\
\text { Modality }\end{array}$ & Findings & ERT & Surgery & $\begin{array}{l}\text { Neurological Symptom } \\
\text { Resolution }\end{array}$ \\
\hline \multirow[t]{2}{*}{1} & \multirow[t]{2}{*}{$3 \mathrm{yrs}, 9 \mathrm{mos}, \mathrm{M}$} & \multirow[t]{2}{*}{$\begin{array}{l}\text { Irritability; nausea/ } \\
\text { vomiting; head- } \\
\text { aches; bilateral } \\
\text { papilledema }\end{array}$} & Head CT & $\begin{array}{l}\text { Left coronal craniosynostosis; sagittal cra- } \\
\text { niosynostosis; widening of right coronal } \\
\text { suture; persistent anterior fontanelle; vol } \\
\text { loss of right frontal lobe; prominent } \\
\text { scalp veins at vertex, skull base, anteri- } \\
\text { or frontal lobe }\end{array}$ & \multirow[t]{2}{*}{ Postop } & \multirow[t]{2}{*}{ Yes } & \multirow[t]{2}{*}{ Yes } \\
\hline & & & Brain MRI & $\begin{array}{l}\text { Vol loss of right frontal lobe; congenital ab- } \\
\text { sence of right sigmoid sinus; multiple } \\
\text { venous anomalies }\end{array}$ & & & \\
\hline \multirow[t]{2}{*}{2} & \multirow[t]{2}{*}{$5 \mathrm{yrs}, 6 \mathrm{mos}, \mathrm{F}$} & \multirow{2}{*}{$\begin{array}{l}\text { Headaches; nystag- } \\
\text { mus; decreased vi- } \\
\text { sual acuity; micro- } \\
\text { cephaly }\end{array}$} & Head CT & $\begin{array}{l}\text { Scalloping of inner table of skull; absence } \\
\text { of extraaxial spaces; left coronal cra- } \\
\text { niosynostosis; sagittal craniosynostosis }\end{array}$ & \multirow[t]{2}{*}{ Postop } & \multirow[t]{2}{*}{ Yes } & \multirow[t]{2}{*}{ Yes } \\
\hline & & & Brain MRI & Dysmorphic calvaria & & & \\
\hline 3 & 8 mos, $\mathrm{F}$ & $\begin{array}{l}\text { Brachycephaly; ventila- } \\
\text { tor dependence }\end{array}$ & Head CT & Bilateral coronal craniosynostosis & Preop & Yes & Pending \\
\hline 4 & 22 mos, $F$ & $\begin{array}{l}\text { Bilateral optic nerve } \\
\text { edema }\end{array}$ & Head CT & $\begin{array}{l}\text { Left coronal craniosynostosis; sagittal cra- } \\
\text { niosynostosis; metopic synostosis }\end{array}$ & Postop & Yes & Yes \\
\hline
\end{tabular}

symptoms attributed to increased ICP including headache, unexplained irritability, and vomiting. This patient showed improvement in his body bone mineralization, tooth eruption, increased weight gain, and overall health. The cranial vault remodeling procedure resulted in increased space in the basal cisterns and foramen magnum with less crowding at the craniovertebral junction (Fig. 2).

\section{Case 2}

The 5-year-and-6-month-old sister of the previously presented male patient presented to our craniofacial team with concerns of head shape, headaches, nystagmus, chronic optic nerve edema, and decreased visual acuity. She was previously evaluated in Saudi Arabia, and concerns were raised regarding her genetic predisposition to HPP and the presence of a complex craniosynostosis. She was subsequently referred to our institution for HPP treatment with asfotase alfa. On presentation, the patient's head circumference was $47 \mathrm{~cm}$ (microcephalic for her age), with a significant bony prominence over the bregma with persistence of an open anterior fontanelle that was tense on palpation. 3D head CT scanning revealed abnormal calvarial morphology with marked scalloping of the inner table with absence of the extraaxial spaces, and complete obliteration of the sagittal and left coronal suture lines. These findings were concerning for chronically elevated ICP (Fig. 3A and B).

Given the chronic changes secondary to untreated HPP, healing of bone postoperatively was a concern. The patient underwent an open cranial vault reconstruction with identification of multiple areas of calcified dura. She underwent multiple barrel stave osteotomies for cranial vault expansion without any perioperative complications, such as CSF leaks, associated with the calcified dura. Seven months following surgery a 3D head CT scan demonstrated improved bone growth of the calvaria (Fig. 3C). Although radiographically left coronal craniosynostosis was evident, clinically the patient did not exhibit asymmetrical orbital findings of unilateral coronal craniosynostosis, such as harlequin deformity. Therefore, barrel stave osteotomies were completed rather than modification of the frontoorbital complex. This management choice is debatable, and other craniofacial surgeons may opt to include a frontoorbital cranioplasty with the osteotomies, but this was the course chosen by our craniofacial team due to the complexity of the patient and high risk of CSF leak because of the calcified dura. The patient's head circumference increased to $49 \mathrm{~cm}$, approaching the normal curve for her age. Symptoms associated with elevated intracranial pressure resolved, with improvement in her headaches, stabilization of chronic optic nerve edema, and improvement in visual acuity (Fig. 4).
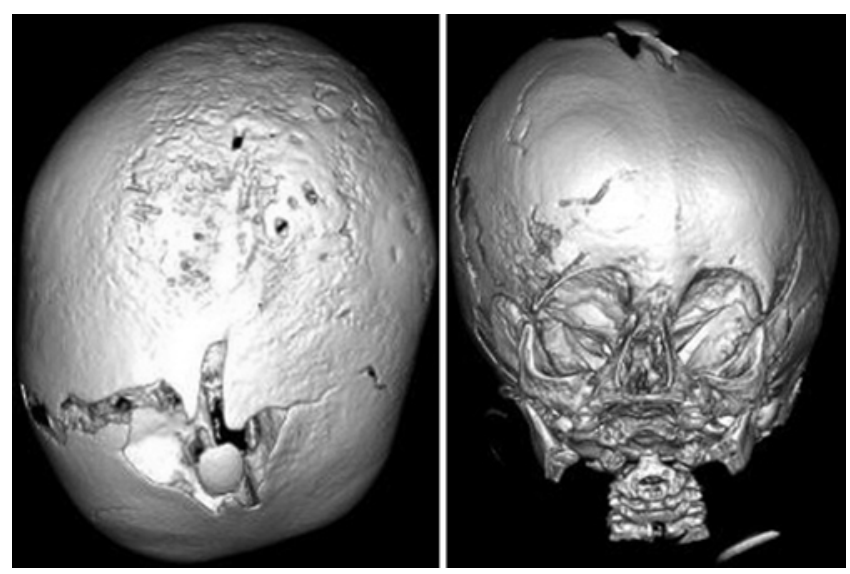

FIG. 1. Case 1. Left: Top view of a 3D head CT scan revealing left coronal and sagittal craniosynostosis with widening of the right coronal suture and persistent anterior fontanelle. Right: Anterior view of a 3D head CT scan revealing left coronal craniosynostosis with facial scoliosis. 


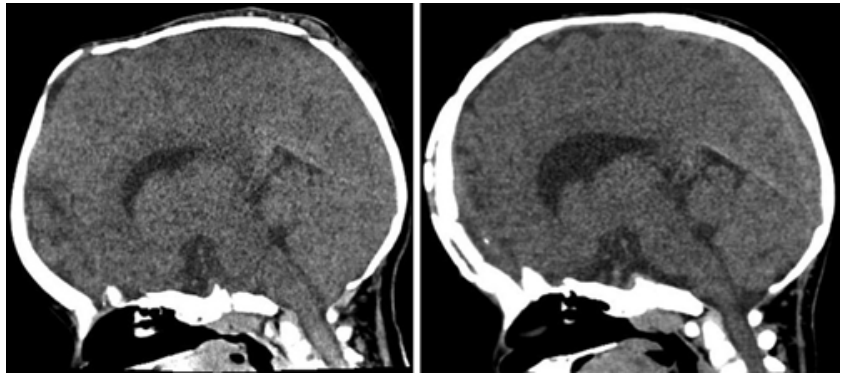

FIG. 2. Case 1. Left: Preoperative sagittal CT scan showing loss of sulci and gyri, scalloping of the inner table, absence of extraaxial spaces, and crowding at the foramen magnum suggestive of Chiari Type I malformation. Right: Postoperative sagittal CT now exhibiting definition of sulci and gyri, increased space in the basal cisterns and extraaxial spaces, and less crowding at the craniovertebral junction.

\section{Discussion}

The inheritance pattern for HPP in the perinatal and infantile forms is autosomal recessive, whereas milder forms may be autosomal dominant or recessive. ${ }^{14}$ There are currently 218 identified mutations in the gene encoding TNSALP with missense mutations accounting for $78.7 \%$ of these mutations. The remaining are microdeletions/insertions $(11.7 \%)$, splicing mutations $(4.8 \%)$, nonsense mutations $(2.7 \%)$, gross deletions $(1.1 \%)$, and nucleotide substitution affecting the major transcription initiation site. ${ }^{14}$

Traditional management of HPP has included symptomatic treatment of the phenotypic manifestations of the disease, including treating hypercalcemia with dietary restriction or calciuretics and orthopedic stabilization of fractures. More recently there have been breakthroughs in treatment of the underlying enzymatic deficiency present in HPP. Various groups have identified that TNSALP activity must be increased in the skeletal bones, and not just in the systemic circulation, to prevent or reverse the pathophysiology of HPP. ${ }^{11}$ Failed attempts to treat HPP with infusions of alkaline phosphatases suggested that TNSALP must be within the skeletal matrix and acting on osteoblasts, chondrocytes, and matrix vesicles to mineralize cartilage and bone. ${ }^{15}$

To treat life-threatening infantile HPP, attempts to
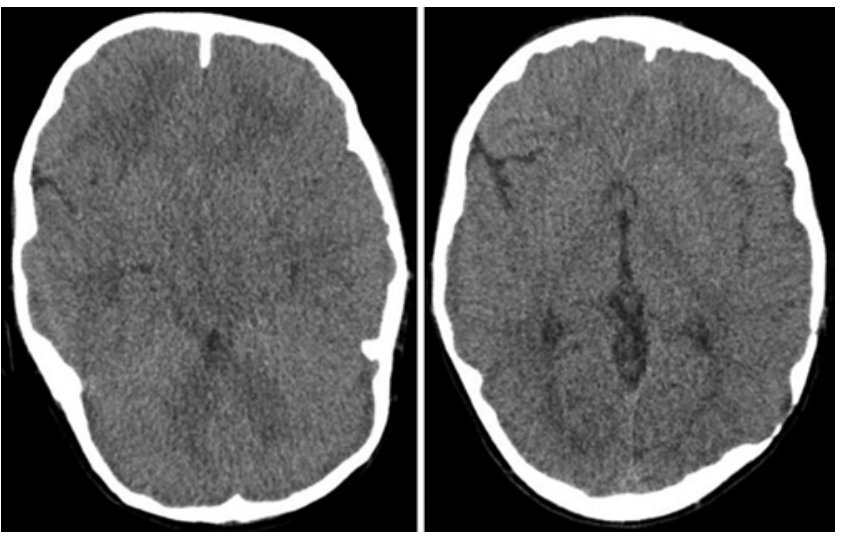

FIG. 4. Case 2. Left: Preoperative axial CT scan demonstrating absence of extraaxial spaces, small ventricular spaces, and tight basilar cisterns. Right: Postoperative axial CT scan revealing cranial expansion, open basal cisterns, reconstitution of the third ventricle, and presence of extraaxial spaces.

transplant rederived mesenchymal stem cells to form TNSALP-replete osteoblasts appeared to benefit 2 female patients but did not provide a definitive cure. ${ }^{2,16}$ Bone-targeted enzyme replacement with the new drug asfotase alfa has previously been effective in infants and young children with life-threatening or severely debilitating HPP. ${ }^{15}$ Asfotase alfa is a recombinant human TNSALP that prevents the manifestations of HPP in TNSALP knockout mice. In a clinical trial of 11 infants and young children with life-threatening HPP, it is associated with improved skeletal radiographs and improved pulmonary and physical function..$^{15}$

The skull appearance in HPP is reported as hypomineralized, and in the severe prenatal form, results in a "caput membranaceum," or soft scalp. In the milder infantile form, widely diastatic sutures or multiple wormian bones are described. 8,10 These features precede the obliteration of sutures and, if seen, should be considered functionally closed sutures. ${ }^{4}$ The premature suture fusion in HPP may start with the sagittal or coronal sutures and progressively involve metopic and lambdoid sutures during childhood. If any of these occurs, intracranial hypertension can develop and the optic nerve should be monitored for development
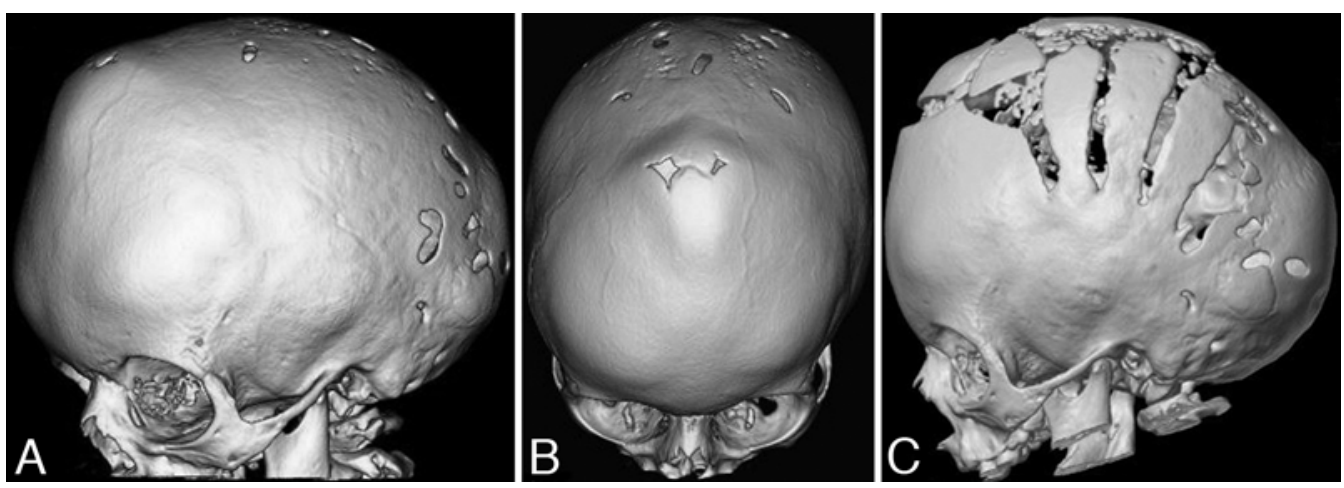

FIG. 3. Case 2. A: Preoperative lateral view of a 3D head CT scan with an obliterated left coronal suture. B: Preoperative top view of a 3D head CT scan with an obliterated sagittal suture and bony prominence over the bregma with persistent anterior fontanelle. C: Postoperative lateral view of a 3D head CT scan demonstrating improved bone growth in the calvaria. 
of papilledema. The lambdoid suture tends to close early in HPP, thus preventing normal growth of the posterior fossa and potentially causing herniation of the cerebellar tonsils with a resulting Chiari Type I malformation. ${ }^{4}$ As seen in Case 1 (Fig. 2), the Chiari Type I malformation can resolve following cranial vault remodeling and expansion, a phenomenon that is described in other forms of craniosynostosis. ${ }^{3,12}$

In the setting of craniosynostosis, CSF outflow can be reduced with impaired CSF absorption secondary to venous sinus hypertension. With the skull unable to expand in craniosynostosis, intracranial hypertension occurs. ${ }^{5}$ The goal of surgical treatment of HPP-related craniosynostosis is to substantially increase the intracranial volume, thereby decreasing ICP. As seen in our 2 case presentations, signs and symptoms associated with suspected elevated ICP (papilledema, headaches, and emesis) can be reversed with cranial volume augmentation.

Enzyme replacement therapy has improved patient outcomes for those children affected by HPP and has now made it possible to diagnose and surgically treat the secondary effects of the disease, such as craniosynostosis. In our case series, all 4 patients received enzyme treatment along with their surgical correction of craniosynostosis (Table 2). In 1 child the enzyme treatment preceded surgery, and in the 3 other patients it followed cranial remodeling. The outcomes from these 2 approaches were similar, and the patients did not have surgical complications. Calvarial reconstitution was excellent in the postoperative period for all 4 patients. We are awaiting a longer followup period for the patient in Case 3 in order to adequately assess resolution of neurological symptoms. These results suggest that surgical correction of HPP-associated craniosynostosis is safe and effective in the setting of ERT. We have found that regardless of the timing of enzyme treatment, the bone in these children is abnormal and trabeculated at the time of surgery. Further study of this growing population of patients needs to be completed to delineate the ideal time to start ERT and which specific outcome variables are improved with ERT.

With the rarity of this disorder, there are no established recommendations in the scientific literature as to when to begin ERT in relation to surgery. The decision on when to initiate enzyme treatment may depend on the subtype of HPP. In the severe infantile form, ERT may allow for improved bone mineralization in the calvaria and systemically, thereby allowing the infant to tolerate a later cranial vault remodeling procedure. This is particularly important with the hypomineralization associated with the ribs and thoracic wall and its relation to ventilation. The patients with the infantile form of HPP traditionally would either not survive to surgical intervention, given ventilation concerns, or would be at elevated risk with positioning and manipulation during surgery. These patients should begin ERT early in order for the child to maximally benefit from the results of the therapy. Our patient with infantile HPP (Case 3 in Table 2) received ERT 6 months prior to surgery and continued it throughout the postoperative course.

In the milder childhood subtype, HPP patients may present with neurological signs or symptoms of elevated ICP, and, as a result, surgical intervention is required to augment intracranial volume and prevent additional neu- rological comorbidities. We then recommend starting ERT 2-3 weeks postoperatively to allow for incision healing prior to bone marrow suppression associated with the enzyme treatment protocol. Two patients in our case series who received enzyme treatment following surgery had improved bone development in the remodeling cranium, aiding the surgical correction (Fig. 2).

To avoid HPP-related complications associated with dural ossifications, CSF leaks, and impaired calvarial bone formation, which can significantly complicate the operations, surgical planning for HPP-related craniosynostosis requires the coordinated efforts of a craniofacial team of neurosurgeons and plastic surgeons. ${ }^{4}$ As evidenced by our case series, intracranial volume expansion in HPP can decrease the signs and symptoms associated with increased ICP and substantially decrease functional risks to the optic nerves by resolving papilledema and to the craniovertebral junction by reducing Chiari Type I malformation. We currently recommend that children undergoing surgery for HPP-related craniosynostosis continue to be monitored throughout childhood and adolescence for the development of Chiari I malformation, syringomyelia, and papilledema.

\section{Conclusions}

With advances in the medical therapy of HPP, patients with infantile and childhood forms of this disease have an improved prognosis. Surgical intervention for treating HPP-related craniosynostosis is crucial to the neurological development of these children by reducing the risk of elevated ICP. With improvements in ERTs, there may be additional children with HPP who become candidates for surgical repair of craniosynostosis. Our case series indicates that these patients may be effectively treated by a craniofacial team familiar with a variety of strategies to reduce the postoperative complications associated with HPP. As more children are successfully treated for HPPrelated craniosynostosis, there is potential for possible prospective trials to further refine treatment strategies and identify the best timing for use of ERT.

\section{References}

1. Baumgartner-Sigl S, Haberlandt E, Mumm S, Scholl-Bürgi S, Sergi C, Ryan L, et al: Pyridoxine-responsive seizures as the first symptom of infantile hypophosphatasia caused by two novel missense mutations (c.677T >C, p.M226T; c.1112C $>$ T, p.T371I) of the tissue-nonspecific alkaline phosphatase gene. Bone 40:1655-1661, 2007

2. Cahill RA, Wenkert D, Perlman SA, Steele A, Coburn SP, McAlister WH, et al: Infantile hypophosphatasia: transplantation therapy trial using bone fragments and cultured osteoblasts. J Clin Endocrinol Metab 92:2923-2930, 2007

3. Cinalli G, Renier D, Sebag G, Sainte-Rose C, Arnaud E, Pierre-Kahn A: Chronic tonsillar herniation in Crouzon's and Apert's syndromes: the role of premature synostosis of the lambdoid suture. J Neurosurg 83:575-582, 1995

4. Collmann H, Mornet E, Gattenlöhner S, Beck C, Girschick $\mathrm{H}$ : Neurosurgical aspects of childhood hypophosphatasia. Childs Nerv Syst 25:217-223, 2009

5. Collmann H, Sörensen N, Krauss J: Hydrocephalus in craniosynostosis: a review. Childs Nerv Syst 21:902-912, 2005

6. Fallon MD, Teitelbaum SL, Weinstein RS, Goldfischer S, 
Brown DM, Whyte MP: Hypophosphatasia: clinicopathologic comparison of the infantile, childhood, and adult forms. Medicine (Baltimore) 63:12-24, 1984

7. Fleisch H, Russell RG, Straumann F: Effect of pyrophosphate on hydroxyapatite and its implications in calcium homeostasis. Nature 212:901-903, 1966

8. Kaplan AM, Albright AL, Zimmerman RA, Rorke LB, Li H, Boyett JM, et al: Brainstem gliomas in children. A Children's Cancer Group review of 119 cases. Pediatr Neurosurg 24:185-192, 1996

9. Litmanovitz L, Reish O, Dolfin T, Arnon S, Regev R, Grinshpan G, et al: Glu274Lys/Gly309Arg mutation of the tissuenonspecific alkaline phosphatase gene in neonatal hypophosphatasia associated with convulsions. J Inherit Metab Dis 25:35-40, 2002

10. Macpherson RI, Kroeker M, Houston CS: Hypophosphatasia. J Can Assoc Radiol 23:16-26, 1972

11. Millán JL, Plotkin H: Hypophosphatasia - pathophysiology and treatment. Actual osteol 8:164-182, 2012

12. Moore MH, Abbott AH, Netherway DJ, Menard R, Hanieh A: Bilambdoid and posterior sagittal synostosis: the Mercedes Benz syndrome. J Craniofac Surg 9:417-422, 1998

13. Mornet E: Hypophosphatasia. Orphanet J Rare Dis 2:40, 2007

14. Mornet E, Nunes ME: Hypophosphatasia, in Pagon RA, Adam MP, Ardinger HH, et al (eds): GeneReviews. Seattle: University of Washington, 2011

15. Wenkert D, McAlister WH, Coburn SP, Zerega JA, Ryan LM, Ericson KL, et al: Hypophosphatasia: nonlethal disease despite skeletal presentation in utero (17 new cases and literature review). J Bone Miner Res 26:2389-2398, 2011
16. Whyte MP, Greenberg CR, Salman NJ, Bober MB, McAlister WH, Wenkert D, et al: Enzyme-replacement therapy in lifethreatening hypophosphatasia. N Engl J Med 366:904-913, 2012

17. Whyte MP, Kurtzberg J, McAlister WH, Mumm S, Podgornik MN, Coburn SP, et al: Marrow cell transplantation for infantile hypophosphatasia. J Bone Miner Res 18:624-636, 2003

\section{Author Contributions}

Conception and design: Kosnik-Infinger, Gordon, Vogel. Acquisition of data: Kosnik-Infinger, Gendron, Vogel. Analysis and interpretation of data: Kosnik-Infinger, Vogel. Drafting the article: Kosnik-Infinger, Gendron, Vogel. Critically revising the article: Kosnik-Infinger, Pan, van Aalst, Vogel. Reviewed submitted version of manuscript: all authors. Approved the final version of the manuscript on behalf of all authors: Kosnik-Infinger. Administrative/technical/material support: Kosnik-Infinger, Vogel. Study supervision: Vogel.

\section{Correspondence}

Libby Kosnik-Infinger, Cincinnati Children's Hospital Medical Center, Division of Pediatric Neurosurgery, MLC 2016, 3333 Burnet Ave., Cincinnati, OH 45229-3026. email: lkinfinger@ gmail.com. 